\title{
论文
}

\section{原位宇宙成因核素深度剖面法解析非稳态 地貌演化}

\author{
杨业, 崔丽峰, 徐胜 ${ }^{*}$, 曹振平, 张顺成, 刘丛强 \\ 天津大学地球系统科学学院表层地球系统科学研究院, 天津 300072 \\ * 通讯作者, E-mail: sheng.xu@tju.edu.cn \\ $\dagger$ 通讯作者, E-mail: liucongqiang@tju.edu.cn
}

收稿日期: 2021-06-04; 收修改稿日期: 2021-09-23; 接受日期: 2021-10-14; 网络版发表日期: 2021-12-20

国家重点研发计划项目(批准号：2020YFA0607700)、天津市研究生科研创新项目(编号：2019YJSB182)和国家自然科学基金项目(批准号: 41930642)资助

摘要原位宇宙成因核素在表层地球系统科学中的应用得到越来越多的关注, 在 $10^{3} \sim 10^{6}$ 年尺度地表剥蚀速率模 型研究中, 稳定剥蚀是一个非常重要的假设. 然而, 如何验证稳定剥蚀的合理性或如何在非稳定剥蚀地区得到可 靠的剥蚀速率成为了研究难点。宇宙成因核素深度剖面法已经被广泛应用于稳定剥蚀地区沉积物和风化壳剖面, 计算剥蚀速率、暴露年龄和继承浓度。本文通过分析天津蓟县和云南腾冲两个典型地区花岗岩风化壳深度剖面 样品中原位宇宙成因核素 ${ }^{10} \mathrm{Be}$ 和 ${ }^{26} \mathrm{Al}$ 浓度, 使用稳态和非稳态(持续暴露、剥蚀速率变化和短期剥蚀事件)深度剖 面模型解析非稳态地貌演化过程. 结果表明, 两地较差的稳态模型模拟结果拟合度反映花岗岩风化壳地表均处于 非稳态. ${ }^{10} \mathrm{Be}$ 非稳态模型结果显示蓟县剖面在千年时间尺度上经历了剥蚀速率改变或地表剥蚀事件, 造成剖面处 于非稳定状态。剥蚀速率变化和短期剥蚀事件模型相似的速率 $\left(14.6_{-12.9}^{+9.4}\right.$ 和 $\left.14.7_{-14.7}^{+9.0} \mathrm{~mm} \mathrm{kyr}^{-1}\right)$ 可能真实地反映了地 表的长期剥蚀, 而高估的剥蚀速率 $\left(39.0_{-2.3}^{+2.6}\right.$ 和 $\left.39.0_{-2.4}^{+2.5} \mathrm{~mm} \mathrm{kyr}^{-1}\right)$ 可能是由于稳定剥蚀前提假设和忽略继承性浓度所 致. 腾冲剖面非稳态模型结果表明, 在 157ka $\left({ }^{10} \mathrm{Be}\right)$ 或 $127 \mathrm{ka}\left({ }^{26} \mathrm{Al}\right)$ 前风化壳剖面经历了强烈的地表剥蚀, 该剥蚀事 件年龄与Termination II 深海氧同位素阶段MIS 6冰期结束年龄(130ka)及青藏高原地区气候波动事件年龄基本一 致, 可能指示了青藏高原地区由冰期向间冰期转变过程中对地表地貌的强烈改造作用. 与青藏高原流域剥蚀速率 研究结果对比, 显示稳态假设条件下的剥蚀速率与区域构造活动强度成正相关关系, 表明构造活动强烈的非稳态 区域的宇宙成因核素剥蚀速率很可能由于稳态假设而被高估. 因此, 本文建议在构造活动强烈或气候变化明显地 区, 结合使用深度剖面稳态和非稳态模型反演可以获得更为精确和有效的剥蚀速率和暴露年龄.

关键词剥蚀速率, 地貌演化, 第四纪定年, 深度剖面, 宇宙成因核素 


\section{1 引言}

自1912年宇宙射线被奥地利物理学家维克托·弗 朗西斯·赫斯(Victor Franz Hess)发现以来, 宇宙成因核 素 ${ }^{3} \mathrm{He} 、{ }^{10} \mathrm{Be} 、{ }^{14} \mathrm{C} 、{ }^{26} \mathrm{Al} 、{ }^{21} \mathrm{Ne}$ 和 ${ }^{36} \mathrm{Cl}$ 等相继被发现并 应用于地球科学领域(Phillips等, 2016). 20世纪70年代, 随着加速器质谱分析技术的出现以及随之测量精度的 不断提高, Lal(1991)把原位(in-situ) 宇宙成因核素从传 统大气(Meteoric)宇宙成因核素中剥离出来, 为研究原 位宇宙成因核素开辟了新的道路. 原位宇宙成因核素 方法学的建立(Lal, 1991; Gosse和Phillips, 2001; Dunai, 2010; Phillips等，2016)为解决冰川暴露定年(Clark等, 2009; Strasky等, 2009)、古人类研究(Shen等, 2009)、 湖泊河流演化(Kong等，2009; Shi等，2017; Yang等, 2019; 杨业等，2021)、土壤及风化壳演化(Ackerer等, 2016; Cui等, 2016; Cui等, 2021)、构造抬升速率(Charreau等, 2017; Delmas等, 2018)、断层滑动速率(Chevalier等, 2017)、古海拔计算(Blard等, 2019)和流域剥蚀 速率(Ouimet等，2009; West等，2014)等表层地球系统 科学问题提供了定量研究的基础. 其中, 模型计算往往 基于稳态剥蚀(Steady-state denudation)假设, 然而最近 研究表明在构造活动强烈(West等，2014；Li等，2017; Wang等, 2017; Zhang等, 2021)、易受剥蚀作用的碳酸 盐岩(Yang等，2020)和冰川作用(Yang等，2019)等地区 多为非稳态剥蚀(Non-steady state denudation). 非稳态 剥蚀对于原位宇宙成因核素应用模型带来了新的挑 战，使得在目前广泛使用原位宇宙成因核素时需要从 不同角度审视研究区是否满足稳态剥蚀模型，抑或如 何使用非稳态模型建立更为精确的年代学信息.

花岗岩风化壳由花岗岩基岩原位风化剥蚀演化而 来，其母岩物质的相对均一性和简单暴露历史等剖面 特征, 是研究原位宇宙成因核素模型的理想场所. 前人 曾通过基岩深度剖面模拟原位宇宙成因核素产率、衰 减系数和表层剥蚀速率等(Braucher等，2003; Braucher 等, 2013; Balco, 2017). 为了进一步研究原位宇宙成因 核素模型中稳态假设的适用性，本文对青藏高原东南 缘构造活动强烈地区 (云南腾冲)和华北平原构造活动 相对稳定地区(天津蓟县)的花岗岩风化壳剖面进行系 统采样(图1a)，根据其 ${ }^{10} \mathrm{Be}$ 和 ${ }^{26} \mathrm{Al}$ 浓度的剖面分布特征 估算风化壳暴露年代和剥蚀速率，结合前人研究结果 讨论非稳态剥蚀对稳态剥蚀假设带来的挑战和新视野.

\section{2 研究区概括及采样信息}

蓟县花岗岩剖面发育在天津市蓟州区盘山花岗岩 岩体上(图1和表1), 位于燕山山脉与华北平原过渡地 带, 属于华北克拉通稳定地区, 现代构造活动较弱. 区 域地貌海拔低, 高差变化较小, 起伏度较低, 年降雨量 较小 $(215 \mathrm{~mm})$, 地表演化相对稳定. 采样点位于山腰 处的采石场剖面, 山坡坡度约 $10^{\circ}$, 土壤层-半风化层-基 岩结构保存完好(图1b). 深度剖面地表至 $900 \mathrm{~cm}$ 处坡度 为 $80^{\circ}, 900 \mathrm{~cm}$ 以下为 $70^{\circ}$, 样品深度通过坡度校正后见 表2. 风化壳发育连续且完整, 为原位风化形成, 具有明 显分层和层间过渡带. 土壤层厚度约 $35 \mathrm{~cm}$, 主要为黏 土矿物和石英, 含少量植物根系; $35 \sim 900 \mathrm{~cm}$ 为半风化 层, 长石和黑云母含量随深度增加风化程度降低, 颜色 由顶部红褐色向深部浅灰色变化; $900 \mathrm{~cm}$ 处半风化层 与基岩分界线明显(图1b). 自地表向 $500 \mathrm{~cm}$ 深部共采集 9 个原位宇宙成因核素样品，用于 ${ }^{10} \mathrm{Be}$ 浓度分析(表2). 利用环刀和排水法分别实测风化壳样品密度(见表2) 和基岩密度 $\left(2.57 \mathrm{~g} \mathrm{~cm}^{-3}\right)$.

腾冲花岗岩采样点位于云南省腾冲县高田村(图1 和表1)，区域分布燕山期和喜山期花岗岩以及石炭系 和二叠系变质岩, 主要受南北向构造带控制( $\mathrm{Li}$ 等, 2020). 样品采自燕山期花岗岩风化壳剖面(图1c), 采 样点位于高山区, 海拔高程变化大(图1a), 起伏度高, 降雨丰沛 $\left(1532 \mathrm{~mm} \mathrm{yr}^{-1}\right)$, 新构造运动强烈. 地表植被 茂密, 多为乔木, 少量草本植物. 风化壳发育连续且完 整, 为原位风化形成, 上部 $70 \mathrm{~cm}$ 为红色土壤层, 植物根 系较多, 原生矿物基本全部风化形成次生黏土矿物, 残 留细小石英颗粒; 下部为全风化-半风化层，颜色由红 褐色逐渐过渡为浅灰色，135 160 cm近水平发育石英 脉(图1c); $660 \mathrm{~cm}$ 剖面深处仍未见基岩. 剖面上共采集4 个样品用于原位宇宙成因核素 ${ }^{10} \mathrm{Be}$ 和 ${ }^{26} \mathrm{Al}$ 浓度分析. 根 据野外观测, 假设土壤密度从表层 $1.50 \mathrm{~g} \mathrm{~cm}^{-3}$ 连续过渡 到深部 $400 \mathrm{~cm}$ 处的 $2.40 \mathrm{~g} \mathrm{~cm}^{-3}$.

\section{3 实验方法及模型计算}

\section{1 样品前处理及加速器质谱 ${ }^{10} \mathrm{Be}$ 和 ${ }^{26} \mathrm{Al}$ 测试}

蓟县和腾冲剖面样品化学前处理分别在天津大学 表层地球系统科学研究院和中国科学院地球化学研究 所完成. 样品首先经过粉碎并篎取 $250 \sim 500 \mu \mathrm{m}$ 直径颗 

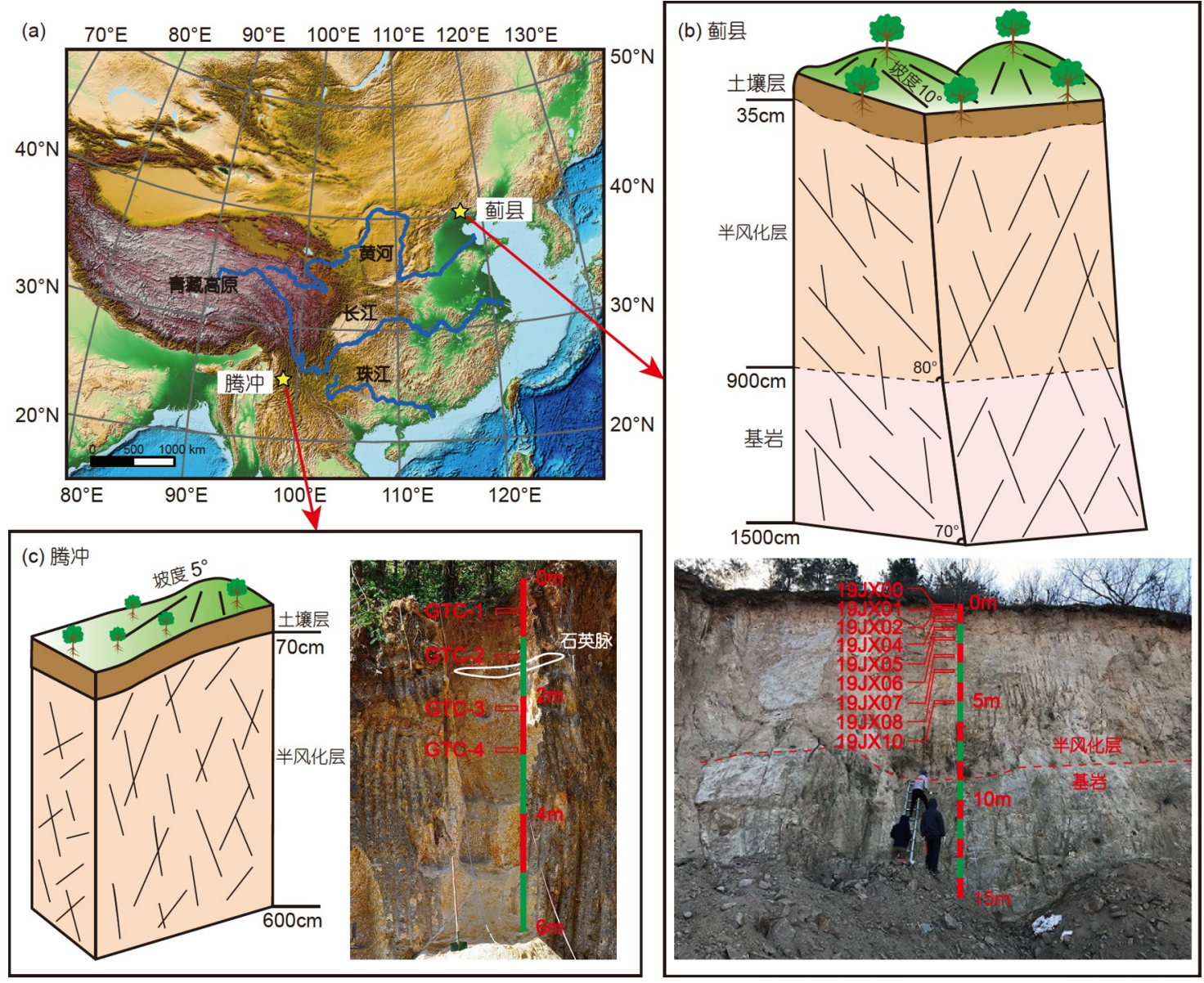

图 1 研究区位置、花岗岩剖面示意图及野外采样信息

粒, 经过磁选去除磁性矿物后用水清洗去除吸附细颗 粒. 分取 $100 \mathrm{~g}$ 样品, 利用过量 $\mathrm{HNO}_{3}$ 淋滤以去除碳酸 盐矿物及颗粒物表面氧化物。使用 $1 \% \mathrm{HF} / \mathrm{HNO}_{3}$ 混合 溶液(去离子水，最后一遍使用 $18.2 \mathrm{M} \Omega$ 超纯水)对样品 进行加热超声刻蚀以去除杂质矿物和颗粒物表面吸 附的大气宇宙成因 ${ }^{10} \mathrm{Be}$ 和 ${ }^{26} \mathrm{Al}$, 多次重复刻蚀获取纯 净石英颗粒(Kohl和Nishiizumi，1992). 利用浓HF消解 $0.5 \mathrm{~g}$ 纯化后的石英，利用电感耦合等离子体光学发射 光谱法(ICP-OES)测试其中 $\mathrm{Al} 、 \mathrm{Fe} 、 \mathrm{Ti}$ 元素含量，其 中 $\mathrm{A} 1$ 含量低于 $150 \mathrm{ppm}$ 为纯净石英的确认标准, 并根 据 $\mathrm{Fe} 、 \mathrm{Ti}$ 含量选择后续处理过程中树脂柱的数量. 称 取 $20 \mathrm{~g}$ 纯净石英样品, 加入 $250 \mu \mathrm{g}$ 的 ${ }^{9} \mathrm{Be}$ 载体和适量 的 ${ }^{27} \mathrm{Al}$ 载体使总 ${ }^{27} \mathrm{Al}$ 含量在 $1.5 \sim 2.0 \mathrm{mg}$, 每克石英样品 加入 $5 \mathrm{~mL}$ 的 $\mathrm{HF}$ 和 $1 \mathrm{~mL}$ 的 $\mathrm{HNO}_{3}$ 进行样品消解并蒸干. 使用“王水”将蒸干样品再次溶解以释放氟化物颗粒 中的 $\mathrm{Al}$, 使之全部进入溶液中. 样品中总 ${ }^{27} \mathrm{Al}$ 含量使用
ICP-OES进行测试分析. 随后, 分别使用阴、阳离子交 换柱分离并提取 $\mathrm{Be}$ 和 $\mathrm{Al}$ ，以氢氧化物形式 $\mathrm{Be}(\mathrm{OH})_{2}$ 和 $\mathrm{Al}(\mathrm{OH})_{3}$ 进行沉淀. 使用马弗炉在 $800^{\circ} \mathrm{C}$ 条件下加热两 小时, 将 $\mathrm{Be}(\mathrm{OH})_{2}$ 和 $\mathrm{Al}(\mathrm{OH})_{3}$ 分别转化为氧化物 $\mathrm{BeO}$ 和 $\mathrm{Al}_{2} \mathrm{O}_{3}$. 最后, 将 $\mathrm{BeO}$ 和 $\mathrm{Al}_{2} \mathrm{O}_{3}$ 分别与铌粉和银粉按重量 比 $\mathrm{BeO}: \mathrm{Nb}=1: 6$ 和 $\mathrm{Al}_{2} \mathrm{O}_{3}: \mathrm{Ag}=1: 3$ 均匀混合后压靶, 分别 装入加速器质谱仪(AMS) 离子源进行 ${ }^{10} \mathrm{Be} /{ }^{9} \mathrm{Be}$ 和 ${ }^{26} \mathrm{Al} /$ ${ }^{27} \mathrm{Al}$ 比值的测试. 蓟县剖面样品 ${ }^{10} \mathrm{Be} /{ }^{9} \mathrm{Be}$ 比值在天津大 学表层地球系统科学研究院 $0.5 \mathrm{MV}$ AMS 上进行测试 (Dong等, 2018), 而腾冲剖面样品 ${ }^{10} \mathrm{Be} /{ }^{9} \mathrm{Be}$ 和 ${ }^{26} \mathrm{Al} /{ }^{27} \mathrm{Al}$ 比值则在英国格拉斯哥大学苏格兰大学联盟环境研 究中心(SUERC)5MV AMS上进行分析(Xu等, 2010). 所有样品的 ${ }^{10} \mathrm{Be} /{ }^{9} \mathrm{Be}$ 和 ${ }^{26} \mathrm{Al} /{ }^{27} \mathrm{Al}$ 比值分别用标准样品

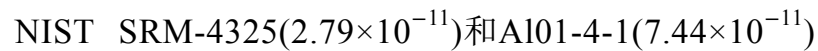
进行归一化, 并进行了化学流程和仪器空白的本底 校正. 
表 1 天津蓟县和云南腾冲花岗岩风化壳剖面样品信息和 模型参数

\begin{tabular}{|c|c|c|}
\hline 采样点 & 蓟县(19JX) & 腾冲(GTC) \\
\hline 纬度 & $40.0404^{\circ} \mathrm{N}$ & $25.1086^{\circ} \mathrm{N}$ \\
\hline 经度 & $117.2948^{\circ} \mathrm{E}$ & $98.3633^{\circ} \mathrm{E}$ \\
\hline 海拔(m) & 82 & 1481 \\
\hline 屏蔽系数 ${ }^{a)}$ & 0.9978 & 0.9780 \\
\hline $\mathrm{P} 10_{\text {spal }}^{\text {b) }}\left(\right.$ at g $\left.^{-1} \mathrm{a}^{-1}\right)$ & 4.0459 & 8.8680 \\
\hline $\mathrm{P} 10_{\text {slow }}{ }^{\mathrm{c})}\left(\right.$ at $\left.\mathrm{g}^{-1} \mathrm{a}^{-1}\right)$ & 0.0997 & 0.1840 \\
\hline $\mathrm{P} 10_{\text {fast }}{ }^{\mathrm{c}}\left(\right.$ at $\left.^{-1} \mathrm{a}^{-1}\right)$ & 0.0462 & 0.0727 \\
\hline $\mathrm{P} 26_{\text {slow }}{ }^{\mathrm{c})}\left(\right.$ at $\left.^{-1} \mathrm{a}^{-1}\right)$ & - & 1.5667 \\
\hline $\mathrm{P} 26_{\text {fast }}{ }^{\mathrm{c}}\left(\right.$ at $\left.\mathrm{g}^{-1} \mathrm{a}^{-1}\right)$ & - & 0.5956 \\
\hline$\Lambda_{\text {slow }}{ }^{\mathrm{d})}\left(\mathrm{g} \mathrm{cm}^{-2}\right)$ & 1155 & 978 \\
\hline$\Lambda_{\text {fast }}^{\mathrm{d})}\left(\mathrm{g} \mathrm{cm}^{-2}\right)$ & 4429 & 3645 \\
\hline
\end{tabular}

a) 根据CRONUS-Earth在线计算器(Balco等，2008); b) 使用 SLHL散裂产率4.01 at g $\mathrm{a} \mathrm{a}^{-1}$ (Borchers等, 2016)和Stone(2000)模型计 算, 见网络版附录代码(http://earthcn.scichina.com); c) 表层 $\mu$ 介子产 率(Balco(2017), 见网络版附录代码; d) 最优拟合衰减长度(Yang等, 2020), 见网络版附录代码

\section{2 模型计算}

石英中原位宇宙成因核素 ${ }^{10} \mathrm{Be}$ 和 ${ }^{26} \mathrm{Al}$ 主要分别 由 ${ }^{16} \mathrm{O}$ 和 ${ }^{28} \mathrm{Si}$ 散裂反应(spallation)、快 $\mu$ 介子(fast muons)
和慢 $\mu$ 介子(slow/negative muons)反应产生(图2a). 宇宙 成因核素的散裂反应产率 $(P(x, \varepsilon, t))$ 随深度 $(x(\varepsilon, t))$ 呈近 似指数型变化:

$P(x, \varepsilon, t)=P_{0} \mathrm{e}^{-\frac{\rho x(\varepsilon, t)}{1}}$,

式中, $P_{0} 、 \varepsilon 、 t 、 \Lambda$ 分别为核素地表产率 $\left(a t \mathrm{~g}^{-1} \mathrm{a}^{-1}\right)$ 、 剥蚀速率 $\left(\mathrm{cm} \mathrm{a}^{-1}\right)$ 、暴露时间(a)和宇宙射线粒子的衰 减长度 $\left(\mathrm{g} \mathrm{cm}^{-2}\right)$. 散裂中子有效衰减长度随着海拔和纬 度变化而变化, 其范围在150 190 $\mathrm{g} \mathrm{cm}^{-2}$ (Gosse和Phillips, 2001; Dunai, 2010; Marrero等, 2016), 本文选取 $160 \mathrm{~g} \mathrm{~cm}^{-2}$. $\mu$ 介子衰减长度相对于散裂中子较长, 其中 慢 $\mu$ 介子和快 $\mu$ 介子衰减长度分别采用蓟县的 1158 和 $4439 \mathrm{~g} \mathrm{~cm}^{-2}$ 以及腾冲的 978 和 $3645 \mathrm{~g} \mathrm{~cm}^{-2}$ (表1)(Yang等, 2020). 如图2a所示, 核素在表层主要由散裂中子反应 产生, 而在深部主要由 $\mu$ 介子反应产生. 宇宙成因核素 浓度累积量 $(C(x, \varepsilon, t))$ 与地表剥蚀速率 $(\varepsilon)$ 和暴露年龄 $(t)$ 方程式如下 (Lal, 1991):

$$
\frac{\mathrm{d} C(x, \varepsilon, t)}{\mathrm{d} t}=-\lambda C(x, \varepsilon, t)+P(x, \varepsilon, t),
$$

式中, ${ }^{10} \mathrm{Be}$ 和 ${ }^{26} \mathrm{Al}$ 衰变常数 $\lambda$ 分别为 $5.00 \times 10^{-7} \mathrm{a}^{-1}$ (Chmeleff等, 2010; Korschinek等, 2010)和 $9.83 \times 10^{-7} \mathrm{a}^{-1}$ (Nishiizumi, 2004). 当地表发生剥蚀并假

表 2 天津蓟县和云南腾冲花岗岩风化壳剖面的原位宇宙成因核素 ${ }^{10} \mathrm{Be}$ 和 ${ }^{26} \mathrm{Al}$ 分析结果

\begin{tabular}{|c|c|c|c|c|c|c|c|c|c|c|c|c|c|c|}
\hline 样品 & $\begin{array}{c}\text { 深度 }^{\mathrm{a})} \\
(\mathrm{cm})\end{array}$ & $\begin{array}{c}\text { 实测密度 }) \\
\left(\mathrm{g} \mathrm{cm}^{-3}\right)\end{array}$ & $\begin{array}{l}\text { 有效密度 } \\
\left(\mathrm{g} \mathrm{cm}^{-3}\right)\end{array}$ & $\begin{array}{c}\text { 石英质量 } \\
(\mathrm{g})\end{array}$ & $\begin{array}{c}{ }^{9} \mathrm{Be} \text { 载体 } \\
(\mu \mathrm{g})\end{array}$ & $\begin{array}{c}{ }^{27} \mathrm{Al} \text { 总含 } \\
\text { 量 }(\mu \mathrm{g})\end{array}$ & $\begin{array}{l}{ }^{10} \mathrm{Be} /{ }^{9} \mathrm{Be} \\
\left(\times 10^{-13}\right)\end{array}$ & $\begin{array}{c} \pm \\
(\%) \\
\end{array}$ & $\begin{array}{c}{\left[{ }^{10} \mathrm{Be}\right]} \\
\left(\times 10^{4} \text { at g }^{-1}\right) \\
\end{array}$ & $\begin{array}{c} \pm \\
(\%) \\
\end{array}$ & $\begin{array}{c}{ }^{26} \mathrm{Al} /{ }^{27} \mathrm{Al} \\
\left(\times 10^{-13}\right)\end{array}$ & $\begin{array}{c} \pm \\
(\%)\end{array}$ & $\begin{array}{c}{\left[{ }^{26} \mathrm{Al}\right]} \\
\left(\times 10^{4} \mathrm{at} \mathrm{g}^{-1}\right) \\
\end{array}$ & $\begin{array}{c} \pm \\
(\%) \\
\end{array}$ \\
\hline \multicolumn{15}{|l|}{ 蓟县剖面 } \\
\hline 19JX00 & 5 & 1.40 & 1.40 & 25.953 & 250.4 & - & 1.83 & 6.4 & 14.5 & 6.4 & - & - & - & - \\
\hline 19JX01 & 34 & 1.40 & 1.42 & 27.462 & 250.2 & - & 1.41 & 9.0 & 11.4 & 9.0 & - & - & - & - \\
\hline 19JX02 & 49 & - & 1.43 & 29.597 & 250.7 & - & 1.64 & 7.0 & 11.8 & 7.0 & - & - & - & - \\
\hline 19JX04 & 79 & 1.59 & 1.45 & 28.199 & 249.7 & - & 1.29 & 7.7 & 10.5 & 7.7 & - & - & - & - \\
\hline 19JX05 & 118 & - & 1.48 & 27.250 & 250.1 & - & 0.82 & 10.1 & 8.2 & 10.1 & - & - & - & - \\
\hline 19JX06 & 177 & 1.60 & 1.52 & 27.953 & 249.1 & - & 0.76 & 11.8 & 7.7 & 11.8 & - & - & - & - \\
\hline 19JX07 & 256 & 1.70 & 1.57 & 26.921 & 249.1 & - & 0.68 & 49.1 & 7.5 & 49.1 & - & - & - & - \\
\hline 19JX08 & 335 & 1.85 & 1.62 & 29.508 & 249.8 & - & 0.36 & 21.9 & 5.3 & 21.9 & - & - & - & - \\
\hline 19JX10 & 492 & - & 1.72 & 29.910 & 250.0 & - & 0.55 & 20.1 & 6.1 & 20.1 & - & - & - & - \\
\hline \multicolumn{15}{|l|}{ 腾冲剖面 } \\
\hline GTC-1 & 55 & - & 1.56 & 21.635 & 225.9 & 2077 & 4.98 & 2.3 & 34.2 & 2.3 & 10.45 & 1.5 & 223.0 & 1.5 \\
\hline GTC-2 & 135 & - & 1.65 & 22.638 & 225.5 & 2534 & 2.32 & 2.4 & 15.0 & 2.4 & 4.07 & 2.2 & 103.5 & 2.2 \\
\hline GTC-3 & 225 & - & 1.75 & 25.325 & 224.6 & 2355 & 1.07 & 2.2 & 6.1 & 2.2 & 2.07 & 2.4 & 42.3 & 2.4 \\
\hline GTC-4 & 295 & - & 1.83 & 16.505 & 221.7 & 1383 & 0.47 & 3.2 & 4.0 & 3.2 & 1.39 & 3.1 & 26.5 & 3.1 \\
\hline
\end{tabular}

a) 根据地表及剖面坡度校正后深度, 误差均为 $\pm 2.5 \mathrm{~cm}$. b) 蓟县和腾冲剖面地表密度分别为 1.40 和 $1.50 \mathrm{~g} \mathrm{~cm}^{-3}$ 


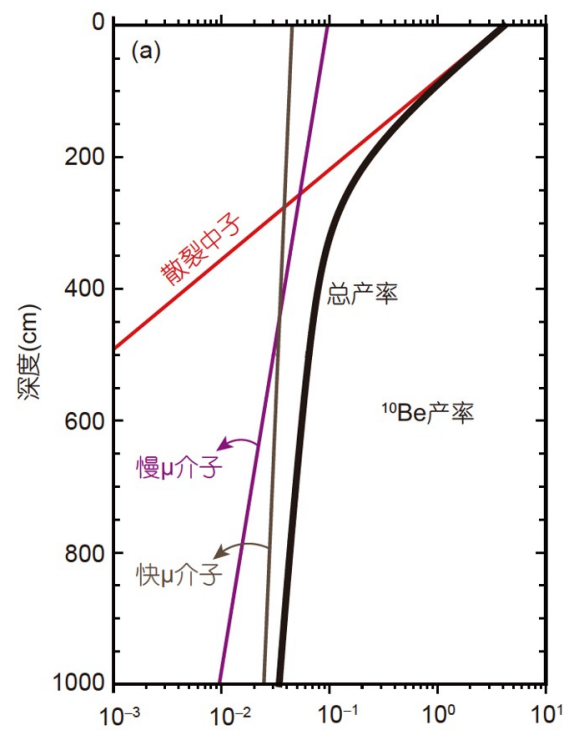

(b)

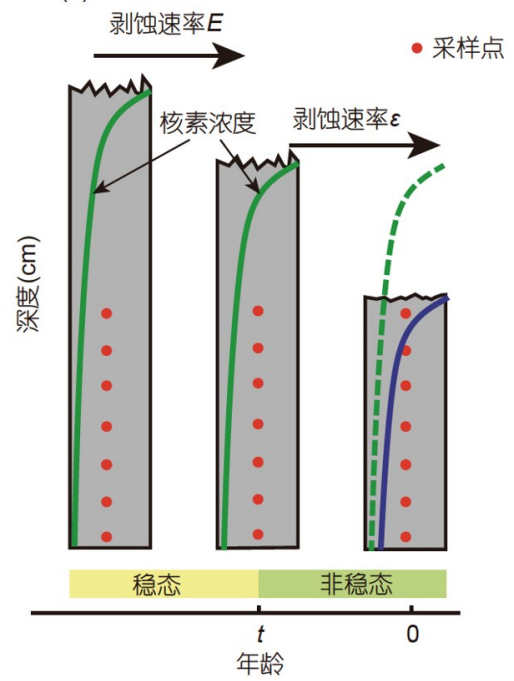

(c)

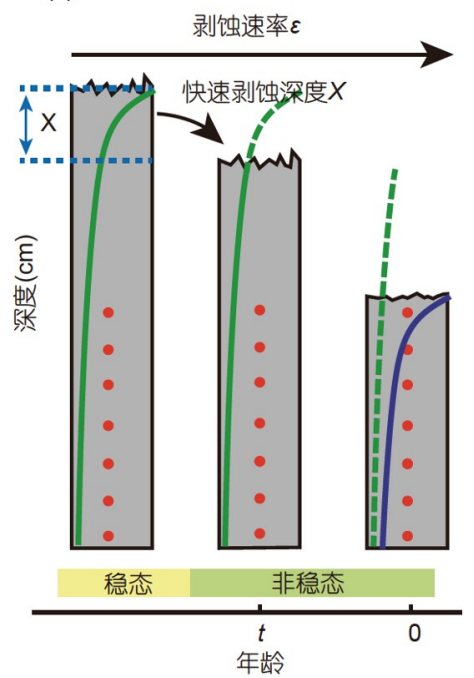

图 2 原位宇宙成因核素 ${ }^{10} \mathrm{Be}$ 产率随深度变化 $(\mathbf{a})$ 、剥蚀速率变化模型由稳定剥蚀速率 $E$ 改变为剥蚀速率 $\varepsilon(\mathbf{b})$ 和短期剥蚀事件 模型造成剥蚀深度 $X(\mathbf{c})$ 示意图

(a) 表明核素产率表层主要受散裂反应(96.6\%)控制, 而在深部主要受 $\mu$ 介子控制. 其中密度 $\rho$ 为 $2.7 \mathrm{~g} \mathrm{~cm}^{-3}$, 产率及衰减长度采用SLHL参数. (b), (c) 绿色虚线表示继承浓度, 蓝色线表示现在剖面核素浓度, 据Yang等(2020)

设剥蚀速率稳定不变, 样品深度随时间变化如下:

$x(\varepsilon, t)=\xi-\varepsilon t$,

式中， $\xi$ 表示初始深度. 从上述三个方程式求解并使用 式(3)代换去除 $\xi$ 可以得到如下方程式:

$$
\begin{aligned}
C(x, \varepsilon, t)= & C(x, 0) \mathrm{e}^{-\lambda t} \\
& +\frac{P}{\lambda+\frac{\rho}{\Lambda} \varepsilon} \mathrm{e}^{-\frac{\rho}{\Lambda} x}\left[1-\mathrm{e}^{-\left(\lambda+\frac{\rho}{\Lambda} \varepsilon\right) t}\right] .
\end{aligned}
$$

若同时考虑散裂、慢 $\mu$ 介子和快 $\mu$ 介子三种不同 宇宙成因核素的贡献, 且近似 $\mu$ 介子产率随深度呈指数 变化, 那么宇宙成因核素的浓度可以表述为(Braucher 等, 2009; Yang等, 2019):

$$
\begin{aligned}
C(x, \varepsilon, t)= & C(x, 0) \mathrm{e}^{-\lambda t} \\
& +\sum_{i=\text { spal,slow,fast }} \frac{P_{i}}{\lambda+\frac{\rho}{\Lambda_{i}} \varepsilon} \mathrm{e}^{-\frac{\rho}{\Lambda_{i}} x}\left[1-\mathrm{e}^{-\left(\lambda+\frac{\rho}{\Lambda_{i}} \varepsilon\right) t}\right],
\end{aligned}
$$

其中 $C(x, 0)$ 是核素在 $x$ 深度 $(\mathrm{cm})$ 的继承浓度. 原位宇宙 成因核素地表产率受海拔(大气压)和纬度影响，本文 采用4.01 at $\mathrm{g}^{-1} \mathrm{a}^{-1}$ (Borchers等, 2016)为海平面和高纬 度(sea level and high latitude, SLHL)参考面的散裂中子 成因 ${ }^{10} \mathrm{Be}$ 产率，散裂中子 ${ }^{26} \mathrm{Al} /{ }^{10} \mathrm{Be}$ 比值为6.75(Balco等,
2008). 本研究中散裂中子成因 ${ }^{10}$ Be地表产率根据地理 纬度和大气压参数进行校正(表1)(Lal，1991；Stone, 2000), $\mu$ 介子产率根据大气压参数进行校正(表1)(Balco，2017). 地表剥蚀和风化作用造成深度样品密度随 时间变化(Rodés和Evans, 2020), 考虑到花岗岩地表剥 蚀速率缓慢, 故假设在万年有效暴露时间内密度未发 生变化. 本文结合野外实测密度并假设蓟县剖面从表 层的实测密度 $1.40 \mathrm{~g} \mathrm{~cm}^{-3}$ 均匀增加至 $900 \mathrm{~cm}$ 基岩实测 深度的 $2.57 \mathrm{~g} \mathrm{~cm}^{-3}$, 腾冲剖面从表层的 $1.50 \mathrm{~g} \mathrm{~cm}^{-3}$ 到 $400 \mathrm{~cm}$ 深度的 $2.40 \mathrm{~g} \mathrm{~cm}^{-3}$, 并将地表至各样品深度的密 度中值作为全岩有效密度(表2). 相关模型使用Mathematica $^{\mathrm{TM}}$ 进行编译和计算, 详细代码请见网络版附录.

\subsection{1 稳态模型(Model 1)}

稳态一般指剖面经历长时间 $(t>>1 /(\lambda+\rho \varepsilon / \Lambda))$ 的稳 定剥蚀后达到的一种状态, 由式(5)可得到稳态模型, 用于计算地表剥蚀速率:

$C(\varepsilon)=\sum_{i=\text { spal,slow,fast }} \frac{P_{i}}{\lambda+\frac{\rho}{\Lambda_{i}} \varepsilon} \mathrm{e}^{-\frac{\rho}{\Lambda_{i}} x}$,

当样品来自表层 $(x=0)$, 假设剥蚀速率可忽略不计且没 有继承性浓度, 由式(5)可得简单暴露年龄计算模型: 
$C(t)=\sum_{i=\text { spal,slow,fast }} \frac{P_{i}}{\lambda}\left(1-\mathrm{e}^{-\lambda t}\right)$,

其中表层 $\mu$ 介子的产率贡献一般小于 $3.4 \%$ (图2a).

\subsection{2 非稳态模型}

稳态需要地表长时间的稳定连续暴露, 然而在构 造活动不稳定和气候多变地区很难达到稳定状态. 在 地质历史时期，地表往往经历过突发性剥蚀事件或剥 蚀速率改变. 为了模拟最后一次的剥蚀或剥蚀速率变 化, Yang等(2020)提出了剥蚀速率变化(Model 3a) 和短 期剥蚀事件(Model 3b)两种模型(图2b和2c)讨论碳酸 盐岩地区非稳态地貌．本文增加持续暴露模型(Model 2)以讨论继承浓度的重要性.

( i ) 持续暴露模型(Model 2). 假设样品经历稳定 的剥蚀速率和暴露历史, 且忽略继承性浓度. 由式(5) 可得

$$
\begin{aligned}
C(x, \varepsilon, t)= & \sum_{i=\text { spal,slow,fast }} \frac{P_{i}}{\lambda+\frac{\rho}{\Lambda_{i}} \varepsilon} \\
& \times \mathrm{e}^{-\frac{\rho}{\Lambda_{i}} x}\left(1-\mathrm{e}^{-\left(\lambda+\frac{\rho}{\Lambda_{i}}\right) t}\right) .
\end{aligned}
$$

(ii) 剥蚀速率变化模型(Model 3a). 气候、构造及 生态系统的变化事件往往会改变地表的剥蚀速率, 为 了研究最后一次 $(t)$ 事件导致的剥蚀速率变化, 我们假 设剖面由末次事件发生前的稳定剥蚀速率 $E$ 改变为末 次事件发生后的剥蚀速率 $\varepsilon$ (图 $2 \mathrm{~b}$ ), 且考虑剥蚀速率 $E$ 状态下所保留的继承浓度(图 $2 \mathrm{~b}$, 绿色曲线). 由稳态剥 蚀速率方程式(6)作为继承浓度带入方程(5)可得(Yang 等, 2020):

$$
\begin{aligned}
C(x, \varepsilon, t, E)= & \left(\sum_{i=\text { spal,slow,fast }} \frac{P_{i}}{\lambda+\frac{\rho}{\Lambda_{i}} E} \mathrm{e}^{-\frac{\rho}{\Lambda_{i}}(x+\varepsilon t)}\right) \mathrm{e}^{-\lambda t} \\
& +\sum_{i=\text { spal,slow,fast }} \frac{P_{i}}{\lambda+\frac{\rho}{\Lambda_{i}} \varepsilon} \mathrm{e}^{-\frac{\rho}{\Lambda_{i}} x}\left[1-\mathrm{e}^{-\left(\lambda+\frac{\rho}{\Lambda_{i}} \varepsilon\right) t} .\right.
\end{aligned}
$$

(iii) 短期剥蚀事件模型(Model 3b). 短期剥蚀事件 往往会造成地表在短时间内被剥蚀数米甚至数十米, 例如各种崩塌、滑坡或泥石流事件. 这种极端事件往 往会造成地表的突然破坏而形成非稳态地貌. 假设表 层剥蚀速率不变且极端事件前为稳定状态，极端事件
造成的剥蚀深度 $(X)$ 、剥蚀事件年龄 $(t)$ 和剥蚀速率 $(\varepsilon)$ 关系如下(图2c) (Yang等, 2020):

$$
\begin{aligned}
C(x, \varepsilon, t, X)= & \left(\sum_{i=\text { spal,slow,fast }} \frac{P_{i}}{\lambda+\frac{\rho}{\Lambda_{i}} \varepsilon} \mathrm{e}^{-\frac{\rho}{\Lambda_{i}}(x+\varepsilon t+X)}\right) \mathrm{e}^{-\lambda t} \\
& +\sum_{i=\text { spal,slow,fast }} \frac{P_{i}}{\lambda+\frac{\rho}{\Lambda_{i}} \varepsilon} \mathrm{e}^{-\frac{\rho}{\Lambda_{i}} x}\left[1-\mathrm{e}^{-\left(\lambda+\frac{\rho}{\Lambda_{i}} \varepsilon\right) t} .\right.
\end{aligned}
$$

\section{3 卡方最优拟合}

在式(5)、(9)和(10)中都有三个未知参数 $(\varepsilon, t, C$ $(x, 0) / E / X)$. 根据不同深度样品核素浓度, 可以进行卡 方拟合求解最优参数解及置信区间(Stone等, 1998; Siame等, 2004; Wolkowinsky和Granger, 2004; Braucher等, 2009; Hidy等, 2010; Rodés等, 2011; Yang等, 2019):

$\chi^{2}=\sum_{i=1}^{n}\left(\frac{C_{i}-C(x, \varepsilon, t)}{\sigma_{C_{i}}}\right)^{2}$,

其中 $C_{i}$ 和 $\sigma_{C i}$ 分别是 $x$ 深度样品核素浓度和浓度误差, $C$ $(x, \varepsilon, t)$ 是方程模拟的核素浓度. 仅用测量浓度数据很 难同时去模拟不同参数带来的误差影响(Borchers等, 2016), 所以忽略了产率、衰变系数、衰减长度、密度 和深度误差在最优拟合中的误差传递. 其中 ${ }^{10} \mathrm{Be}$ 和 ${ }^{26} \mathrm{Al}$ 产率的误差为 $7.2 \%$ 和 10\%(Phillips等, 2016). 密度误 差也会给深度剖面法造成一定的影响(Rodés等, 2011), 实际计算时, 采用实测密度并考虑密度随深度的变化, 从而减少使用假设单一密度值所带来的误差. 在计算 模型中, 前人基于卡方检验反演(Siame等, 2004; Braucher等, 2009; Rodés等, 2011; Yang等, 2019)、蒙特卡 罗(Hidy等, 2010)或贝叶斯模型(Parnell等, 2011; Marrero等, 2016)均可以求解出 $C(x, 0)$ - $\varepsilon-t$ 最优解及置信区 间. 本文采用三维图形可视化模型(Yang等，2019)来约 束三个参数的最优值及 $1 \sigma$ 置信区间. 相应Mathematica ${ }^{\mathrm{TM}}$ 模型代码计算见网络版附录.

\section{4 结果与讨论}

\section{1 模拟结果及其意义}

蓟县剖面样品中宇宙成因核素 ${ }^{10} \mathrm{Be}$ 浓度变化范围 
为5.3×10 $\sim 14.5 \times 10^{4} \mathrm{at} \mathrm{g}^{-1}$ (表2), 从地表向深部呈近似 指数减少(图3). 其中样品 $19 \mathrm{JX} 07$ 较大的误差 $(49.1 \%$ ) 是由于 $A M S$ 测量 ${ }^{9} \mathrm{Be}^{+}$束流较小, ${ }^{10} \mathrm{Be}$ 计数较低所致, 而 样品19JX05、06、08和10则因自身 ${ }^{10} \mathrm{Be}$ 浓度较低而造 成误差较大. 对数据进行模拟和分析, 稳态(Model 1)和 持续暴露 (Model 2) 模型得出相同的卡方最小值 $\left(\chi_{\text {min }}^{2}=19.96\right)$ 和近似的剥蚀速率 $\left(39.0_{-2.3}^{+2.6}\right.$ 和 $39.0_{-2.4}^{+2.5} \mathrm{~mm}$ $\left.\mathrm{kyr}^{-1}\right)$, 表明在无继承浓度假设条件下该剖面长时间的 持续暴露(Model 2暴露年龄大于1073ka，图3d因已经 达到稳态而使年龄无法收敛)使剖面已经达到稳定状 态. 然而, 在考虑继承浓度后(Model 3), 剥蚀速率变化 (Model 3a) 和短期剥蚀事件(Model 3b)模型卡方最小值 (分别为 3.50 和 3.46) 明显优于稳态和持续暴露模型(卡 方最小值更低则表明拟合度更高)，从而表明考虑继承 浓度模型结果优于忽略继承浓度模型。在图 $3 \mathrm{a}$ 和 $3 \mathrm{c}$ 中, 剖面深部拟合值明显比实际测量值偏小，造成模型与 实际值之间偏差的主要原因是模型忽略了继承浓度的 可能性. 通过考虑不同情况带来的继承浓度, 模拟结果
明显更优(图 $3 \mathrm{e}$ 和 $3 \mathrm{~g}$ ). 剥蚀速率改变模型可以很好的 约束改变前剥蚀速率 $\left(14.6_{-12.9}^{+9.4} \mathrm{~mm} \mathrm{kyr}^{-1}\right)$, 而改变后剥 蚀速率和速率改变事件年龄(图3f)在模型计算中很难 被同时约束，部分原因是年龄和剥蚀速率会此消彼长 (trade-off)以平衡方程模拟最优值(Hidy等，2010; Delmas等, 2018; Yang等, 2019). 模型结果明显显示后剥蚀 速率随着事件年龄的增加而减小，两者呈现反相关关 系. 在约束条件下(后剥蚀速率小于 $500 \mathrm{~mm} \mathrm{kyr}^{-1}$ 和速 率改变事件年龄小于 $100 \mathrm{ka})$, 可以得到后剥蚀速率和 年龄分别为 $214_{-164}^{+286} \mathrm{~mm} \mathrm{kyr}^{-1}$ 和 $10_{-8}^{+90} \mathrm{ka}$, 表明该剖面由 前剥蚀速率 $14.6_{-12.9}^{+9.4} \mathrm{~mm} \mathrm{kyr}^{-1}$ 在 $10_{-8}^{+90} \mathrm{ka}$ 经历了速率改变 事件而转变为 $214_{-164}^{+286} \mathrm{~mm} \mathrm{kyr}^{-1}$. 短期剥蚀事件模型可 以很好的同时约束剥蚀速率、事件年龄和剥蚀深度 (图3 $\mathrm{g} 、 3 \mathrm{~h}$ 和表 3 ), 结果显示剖面在稳定剥蚀速 率 $14.7_{-14.7}^{+9.0} \mathrm{~mm} \mathrm{kyr}^{-1}$ 条件下, 在 $0_{-0}^{+27.3} \mathrm{ka}$ 时经历 $177_{-77}^{+2323}$ $\mathrm{mm}$ 厚度的剥蚀事件. 两种考虑继承浓度的模型都显 示剖面在千年时间尺度上经历了剥蚀速率的改变或者
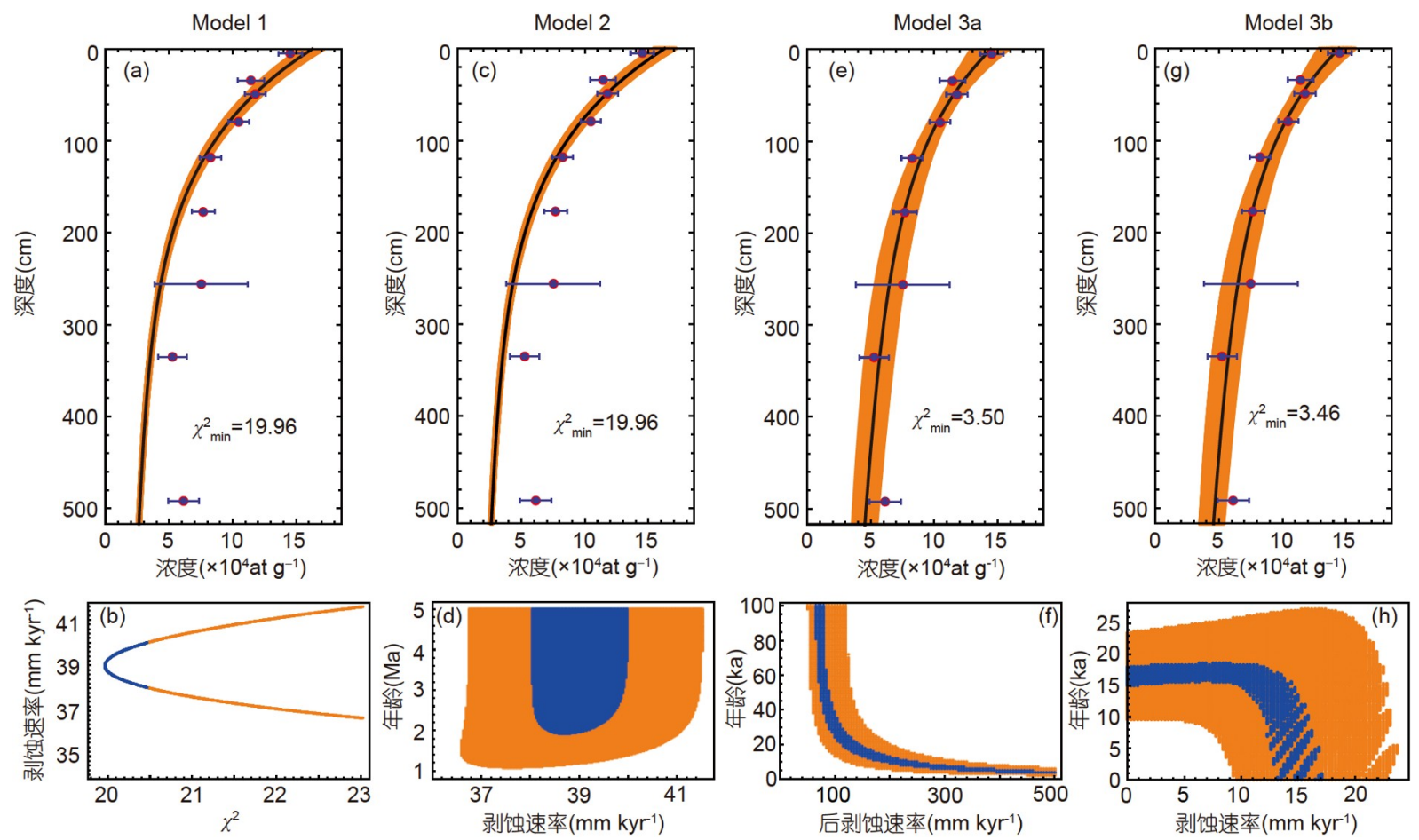

图 3 蓟县 $-{ }^{10} \mathrm{Be}$ 深度剖面稳态 $((\mathrm{a}) 、(\mathrm{~b}))$ 、持续暴露 $((\mathrm{c}) 、(\mathrm{~d}))$ 、剥蚀速率变化 $((\mathrm{e}) 、(\mathrm{f}))$ 和短期剥蚀事件 $((\mathrm{g}) 、(\mathrm{~h}))$ 模型 模拟结果

(a)、(c)、(e)和 $(g)$ 中黑色和橙色曲线分别为深度剖面最优拟合值和 $1 \sigma$ 置信区间. (b)、(d)、(f)和(h)中每个橙色和蓝色点分别代表 $1 \sigma$ 置信区间 和拟合优度小于 $\chi_{\text {min }}^{2}+0.5$ 范围 
杨业等: 原位宇宙成因核素深度剖面法解析非稳态地貌演化

表 3 天津蓟县和云南腾冲花岗岩风化壳剖面的原位宇宙成因核素模拟结果

\begin{tabular}{|c|c|c|c|c|c|c|c|c|c|c|c|c|c|}
\hline \multirow[b]{2}{*}{ 剖面 } & \multicolumn{2}{|c|}{ Model 1} & \multicolumn{3}{|c|}{ Model 2} & \multicolumn{4}{|c|}{ Model 3a } & \multicolumn{4}{|c|}{ Model 3b } \\
\hline & $\chi_{\min }^{2}(-)$ & $\begin{array}{c}\varepsilon \\
\left(\mathrm{mm} \mathrm{kyr}^{-1}\right)\end{array}$ & $\chi_{\min }^{2}(-)$ & $\begin{array}{c}\varepsilon \\
\left(\mathrm{mm} \mathrm{kyr}^{-1}\right)\end{array}$ & $t(\mathrm{ka})$ & $\chi_{\min }^{2}(-)$ & $\left(\mathrm{mm}^{\varepsilon} \mathrm{kyr}^{-1}\right)$ & $t(\mathrm{ka})$ & $\begin{array}{c}E \\
\left(\mathrm{~mm} \mathrm{kyr}^{-1}\right)\end{array}$ & $\chi_{\min }^{2}(-)$ & $\begin{array}{c}\varepsilon \\
\left(\mathrm{mm} \mathrm{kyr}^{-1}\right)\end{array}$ & $t(\mathrm{ka})$ & $X(\mathrm{~mm})$ \\
\hline 蓟县- ${ }^{10} \mathrm{Be}$ & 19.96 & $39.0_{-2.3}^{+2.6}$ & 19.96 & $39.0_{-2.4}^{+2.5}$ & $>1073$ & 3.50 & $214_{-164}^{+286 a)}$ & $10_{-8}^{+90 a)}$ & $14.6_{-12.9}^{+9.4}$ & 3.46 & $14.7_{-14.7}^{+9.0}$ & $0_{-0}^{+27.3}$ & $177_{-77}^{+2323}$ \\
\hline 腾冲_- ${ }^{10} \mathrm{Be}$ & 445.56 & $33.3_{-0.8}^{+0.9}$ & 10.91 & $15.6_{-1.4}^{+1.2}$ & $157_{-20}^{+20}$ & 10.91 & $15.6_{-3.6}^{+1.1}$ & $157_{-57}^{+18}$ & $30 \sim+\infty$ & 10.91 & $15.6_{-2.4}^{+1.2}$ & $157_{-44}^{+19}$ & $5000 \sim+\infty$ \\
\hline 腾冲- ${ }^{26} \mathrm{Al}$ & 699.97 & $30.7_{-0.7}^{+0.7}$ & 4.43 & $14.0_{-1.7}^{+1.3}$ & $127_{-15}^{+15}$ & 4.49 & $13.7_{-12.6}^{+1.3}$ & $121_{-63}^{+16}$ & $238 \sim+\infty$ & 4.43 & $14.0_{-9.8}^{+1.0}$ & $127_{-59}^{+11}$ & $4000 \sim+\infty$ \\
\hline
\end{tabular}

a) 剥蚀速率和事件年龄模拟范围分别为 $0 \sim 500 \mathrm{~mm} \mathrm{kyr}^{-1}$ 和 $0 \sim 100 \mathrm{ka}$

剥蚀事件的发生，造成了剖面在事件之后一直处于非 稳定状态。非稳态模型相似的地表剥蚀速率 $\left(14.6_{-12.9}^{+9.4}\right.$ 和14.7 $\left.{ }_{-14.7}^{+9.0} \mathrm{~mm} \mathrm{kyr}^{-1}\right)$ 可能真实反映了地表的长 期剥蚀速率，而稳态假设条件下百万年长时间尺度的 剥蚀速率 $\left(39.0_{-2.3}^{+2.6}\right.$ 和 $\left.39.0_{-2.4}^{+2.5} \mathrm{~mm} \mathrm{kyr}^{-1}\right)$ 可能由于非稳态 的剥蚀作用而被高估.

腾冲剖面样品宇宙成因核素 ${ }^{10} \mathrm{Be}$ 和 ${ }^{26} \mathrm{Al}$ 浓度变化 范围分别为 $4.0 \times 10^{4} \sim 34.2 \times 10^{4} \mathrm{at} \mathrm{g}^{-1}$ 和 $26.5 \times 10^{4} \sim 223.0 \times 10^{4}$ at $\mathrm{g}^{-1}$ (表2), 两者的浓度从地表向 深部均呈近似指数减少(图4和5). AMS测量过程中离 子束流正常、能量损失低和 ${ }^{10} \mathrm{Be}$ 计数充足保证了样 品 ${ }^{10} \mathrm{Be}$ 浓度误差在 $3.2 \%$ 以内. 通过 ${ }^{10} \mathrm{Be}$ 数据模型分析, 稳态模型异常高的卡方最小值 $\left(\chi_{\min }^{2}=445.56\right.$, 图 $\left.4 b\right)$ 表 明剖面处于非稳态阶段. 三种非稳态模型结果显示一 致的拟合度 $\left(\chi_{\text {min }}^{2}=10.91\right)$, 持续暴露、剥蚀速率变化和 短期剥蚀事件模型剥蚀速率 $(\varepsilon)$ 和年龄 $(t)$ 分别 为 $15.6_{-1.4}^{+1.2} 、 15.6_{-3.6}^{+1.1}$ 和 $15.6_{-2.4}^{+1.2} \mathrm{~mm} \mathrm{kyr}^{-1}$ 和 $157_{-20}^{+20} 、 157_{-57}^{+18}$ 和 $157_{-44}^{+19} \mathrm{ka}$. 剥蚀速率变化模型为前剥蚀速率 $30 \sim$ $+\infty \mathrm{mm} \mathrm{kyr}^{-1}$ 转变为 $15.6_{-3.6}^{+1.1} \mathrm{~mm} \mathrm{kyr}^{-1}$, 短期剥蚀事件模 型经历了 5000 + + mm的剥蚀事件, 结果均表明该事 件发生在 $157 \mathrm{ka}$. 通过 ${ }^{26} \mathrm{Al}$ 数据模型分析, 结果与 ${ }^{10} \mathrm{Be}$ 数据基本一致(表3). 三种非稳态模型结果显示一致的 拟合度 $\left(\chi_{\min }^{2}=4.43 / 4.49 / 4.43\right)$, 持续暴露、剥蚀速率变 化和短期剥蚀事件模型剥蚀速率 $(\varepsilon)$ 和年龄 $(t)$ 分别 为 $14.0_{-1.7}^{+1.3} 、 13.7_{-12.6}^{+1.3}$ 和 $14.0_{-9.8}^{+1.0} \mathrm{~mm} \mathrm{kyr}^{-1}$ 和 $127_{-15}^{+15} 、 121_{-63}^{+16}$ 和127 ${ }_{-59}^{+11} \mathrm{ka}$. 表明在 $127 \mathrm{ka}$ 剖面经历了地表剥蚀或者剥 蚀速率变化事件后一直处于非稳定状态，期间地表平 均剥蚀速率为 $14.0 \mathrm{~mm} \mathrm{kyr}^{-1}$ (表3). 青藏高原的抬升及
区域地形地貌的演化不仅仅与构造活动有关，也与气 候变化密切相关, 例如青藏高原东部末次冰期的退却 造成地貌地表的强烈剥蚀作用(Chevalier和Replumaz, 2019; Yang等, 2019), 晚更新世以来河流阶地的形成与 气候变化密不可分(Pan等, 2009; Yang等, 2019; Tao等, 2020). 腾冲地区剥蚀事件年龄与Termination II 深海氧 同位素阶段MIS 6冰期结束年龄(130ka) (Lisiecki和 Raymo, 2005)基本一致, 可能指示了青藏高原东南缘 地区由冰期向间冰期转变过程中对地表地貌的强烈改 造作用; 同时与青藏高原古里雅冰盖 $132 \mathrm{ka}$ 前 $\delta^{18} \mathrm{O}$ 低分 辨界限(Thompson等，1997)、青藏高原东南地区广泛 分布的冰碛物年龄MIS 6 代表冰川消融后冰碛物开始 暴露时间(Zhou等, 2010; Chevalier和Replumaz, 2019) 和青藏高原内部高海拔湖泊最高古海岸线MIS 5 暴露 年龄(Shi等, 2017; Zhou等, 2020)基本一致, 可能代表 了强烈剥蚀对地表的重塑作用和随后沉积物的堆积 过程.

通过对蓟县和腾冲两个剖面的对比分析, 较差的 稳态模型模拟结果拟合度反映花岗岩风化壳地表均 处于非稳态. 非稳态模型结果显示蓟县剖面在千年时 间尺度上经历了剥蚀速率的改变或地表剥蚀事件, 表 明核素浓度积累主要来自于末次事件前(即模型 3 中 的继承浓度, 图 $2 \mathrm{~b}$ 和 $2 \mathrm{c}$ 绿色虚线). 由于蓟县深度剖面 浓度变化幅度主要受控于事件前的核素浓度积累, 所 以能够较好的约束末次事件前剥蚀速率 $\left(14.6_{-12.9}^{+9.4} \mathrm{~mm}\right.$ $\mathrm{kyr}^{-1}$ ), 但较难约束末次事件后剥蚀速率(表3). 不可忽 略的继承浓度(末次事件前积累核素浓度)造成了 Model 2与 3结果之间的较大差异. 然而, 腾冲剖面非 稳态模型结果表明在 $\sim 157 \mathrm{ka}\left({ }^{10} \mathrm{Be}\right)$ 或 $127 \mathrm{ka}\left({ }^{26} \mathrm{Al}\right)$ 剖 面经历了强烈的地表剥蚀, 其浓度主要形成于末次事 

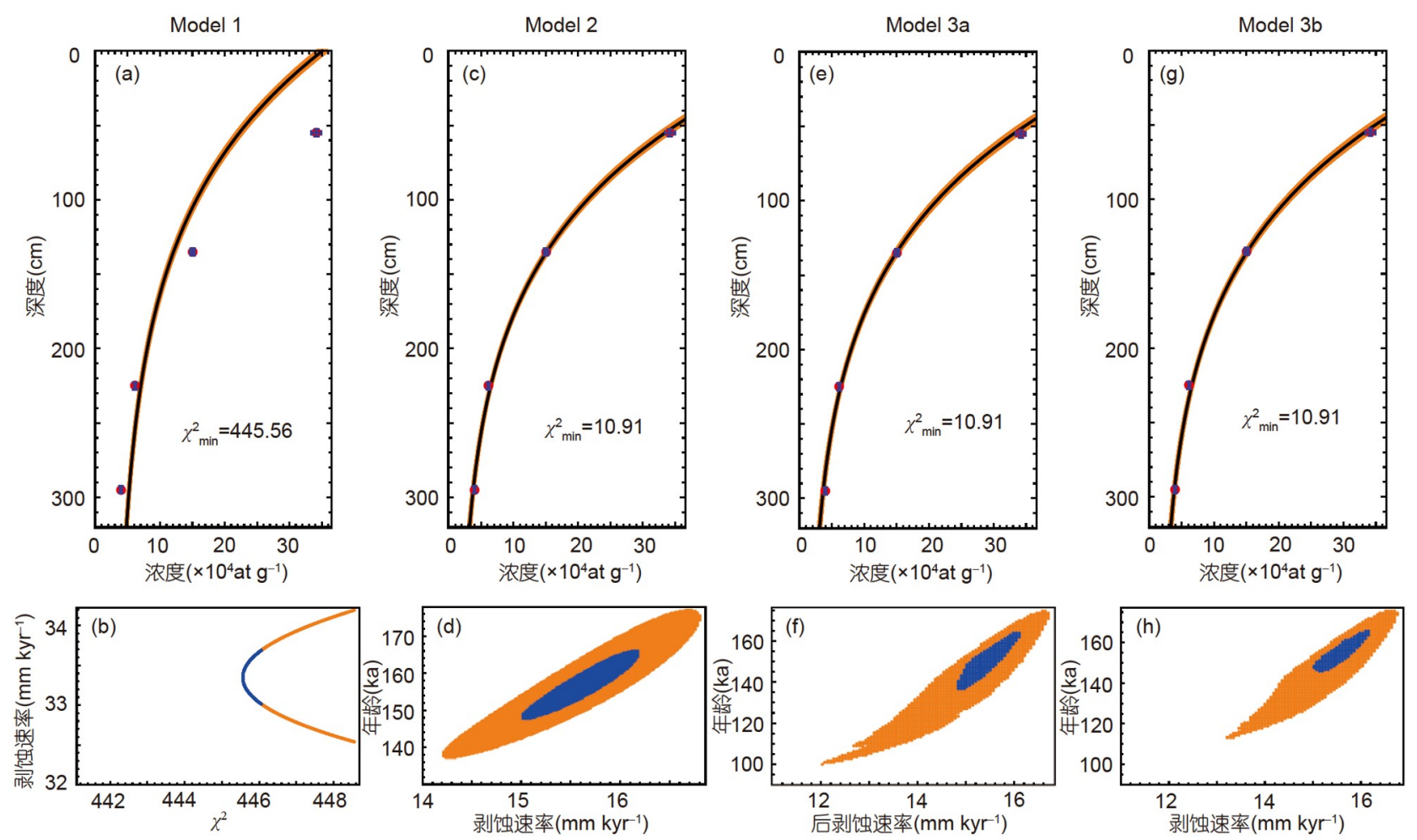

图 4 腾冲 $-{ }^{10} \mathrm{Be}$ 深度剖面稳态 $((\mathrm{a}) 、(\mathrm{~b}))$ 、持续暴露 $((\mathrm{c}) 、(\mathrm{~d}))$ 、剥蚀速率变化 $((\mathrm{e}) 、(\mathrm{f}))$ 和短期剥蚀事件 $((\mathrm{g}) 、(\mathrm{~h}))$ 模型 模拟结果

(a)、(c)、(e)、(g)中黑色和橙色曲线分别为深度剖面最优拟合值和 $1 \sigma$ 置信区间，(b)、(d)、(f)、(h)中每个橙色和蓝色点分别代表 $1 \sigma$ 置信区间 和拟合优度小于 $\chi_{\text {min }}^{2}+0.5$ 范围

件之后，所以能够更好的约束末次事件后剥蚀速率 $\left(15.6_{-3.6}^{+1.1} \mathrm{~mm} \mathrm{kyr}^{-1}\right)$, 但难以约束末次事件前剥蚀速率 (表3). 几乎可以忽略的腾冲剖面继承浓度(末次事件 前积累核素浓度)导致Model 2 与 3 具有相似的结果. 同时, 造成蓟县模拟收玫性较差的原因还有可能是较 高的测量浓度误差和较差的剖面拟合度. 土壤层厚度 受土壤形成速率和表层剥蚀速率的长期共同调节, 其 中土壤形成速率主要受控于基岩岩性、气候、地形 地势和生物扰动等(Heimsath等, 2012). 蓟县花岗岩风 化壳剖面土壤厚度 $(35 \mathrm{~cm})$ 明显低于螣冲花岗岩风化 壳剖面 $(70 \mathrm{~cm})$, 其原因可能是: (1) 腾冲处于较为湿润 的亚热带气候区具有较高的土壤形成速率; (2) 腾冲 土壤自 $157 \mathrm{ka}\left({ }^{10} \mathrm{Be}\right)$ 或 $127 \mathrm{ka}\left({ }^{26} \mathrm{Al}\right)$ 以来稳定发育并 保存完好, 而蓟县土壤剖面千年时间尺度末次事件造 成土壤的丢失.

\section{2 应用稳态模型假设需要注意的问题}

原位宇宙成因核素计算模型的建立(Lal，1991；
Gosse和Phillips，2001; Dunai，2010)为开拓表层地球 系统科学研究提供了新的技术手段, 但其核心计算模 型(式(5))中含有三个自由参数变量: 继承浓度 $\left(C_{0}\right)$ 、 剥蚀速率 $(\varepsilon)$ 和暴露年龄 $(t)$. 通过假设样品已经达到稳 态 $>>1 /(\lambda+\rho \varepsilon / \Lambda)$, 可以分别去除继承浓度和暴露年龄 自由变量得到稳态模型(Model 1). 随着原位宇宙成因 核素研究的不断深入, 诸如阶地沉积物(Hidy等, 2010; Rodés等, 2011; Yang等, 2019)、高山地区现代河流沉 积物(West等, 2014; Wang等, 2017; Zhang等, 2021)等受 构造或气候变化控制的非稳态地区地表剥蚀已经不能 简单地应用稳态模型进行定量分析. 深度剖面法的出 现(Stone等, 1998; Siame等, 2004; Braucher等, 2009; Hidy等, 2010; Rodés等, 2011; Marrero等, 2016; Yang 等, 2019; Yang等, 2020)为同时模拟继承浓度、剥蚀速 率、暴露年龄甚至更为复杂的参数提供了数学模型 支撑.

尽管本文两个花岗岩风化壳剖面均为保存完好 的原位风化壳剖面, 但是模型计算结果显示它们都处 

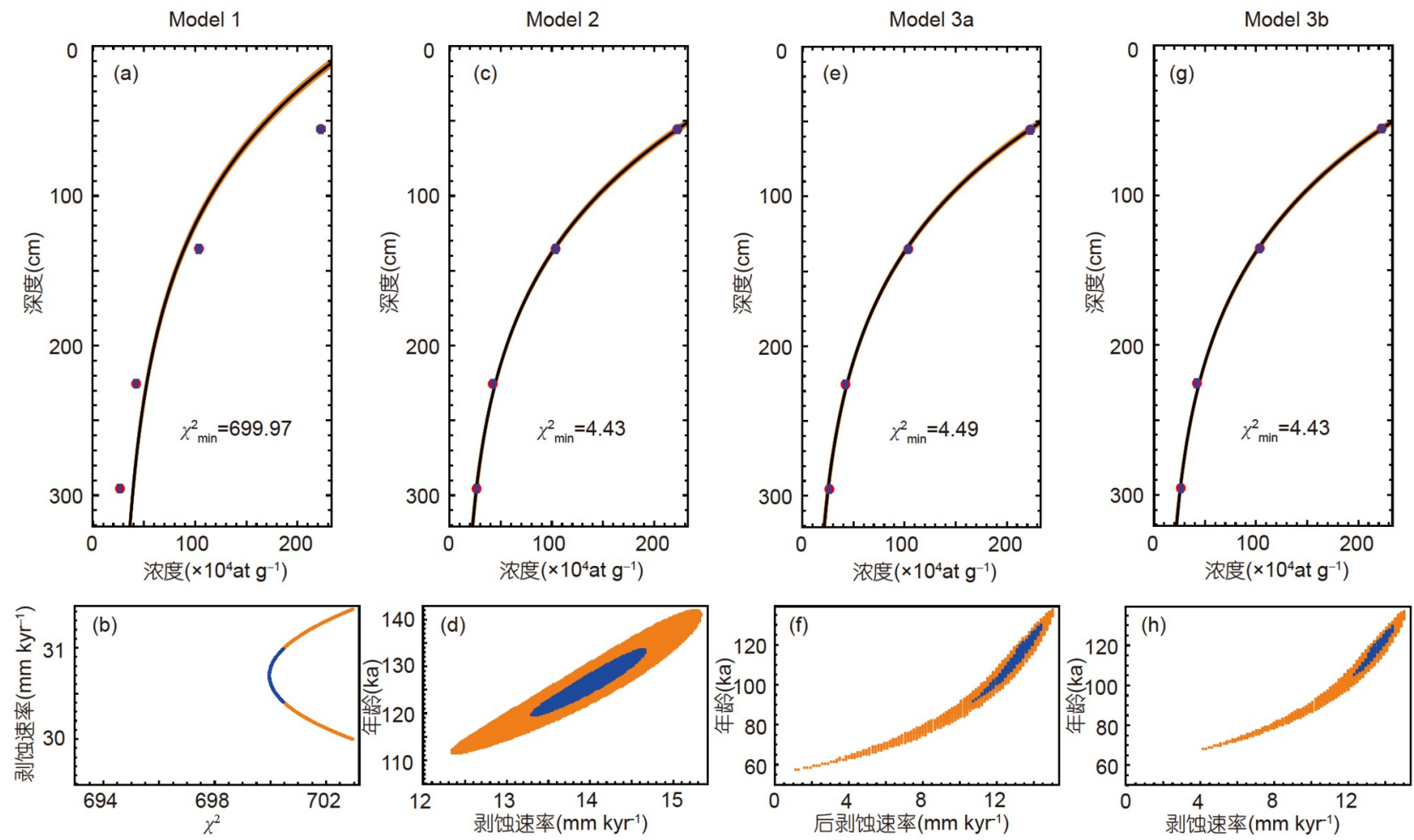

图 5 腾冲 $-{ }_{-}^{26} \mathrm{Al}$ 深度剖面稳态 $((\mathrm{a}) 、(\mathrm{~b}))$ 、持续暴露 $((\mathrm{c}) 、(\mathrm{~d}))$ 、剥蚀速率变化 $((\mathrm{e}) 、(\mathrm{f}))$ 和短期剥蚀事件 $((\mathrm{g}) 、(\mathrm{~h}))$ 模型 模拟结果

(a)、(c)、(e)、(g)中黑色和橙色曲线分别为深度剖面最优拟合值和 $1 \sigma$ 置信区间，(b)、(d)、(f)、(h)中每个橙色和蓝色点分别代表 $1 \sigma$ 置信区间 和拟合优度小于 $\chi_{\min }^{2}+0.5$ 范围

于非稳态阶段，从而表明在地质历史时期由于构造运 动或气候变化带来的地表短期剥蚀事件或剥蚀速率 变化都会破坏地表的稳定性. 青藏高原东北缘黄河流 域剥蚀速率(12 228 $\mathrm{mm} \mathrm{kyr}^{-1}, N=27$ )(Harkins等, 2007; Kirby和Harkins, 2013; Ansberque等, 2015), 东部三江 (怒江、澜沧江、金沙江)及长江支流(雅聋江、大渡 河和岷江)流域剥蚀速率(23 7600 $\left.\mathrm{mm} \mathrm{kyr}^{-1}, N=181\right)$ (Ouimet等, 2009; Godard等, 2010; Henck等, 2011; Ansberque等, 2015; Cook等, 2018)和南部雅鲁藏布江 流域剥蚀速率(41 4416 mm kyr ${ }^{-1}, N=81$ )(Lupker等, 2017; Zhang等, 2021)的统计分析结果显示 ${ }^{10} \mathrm{Be}$ 剥蚀 速率明显受地形特征影响(图6a)。在青藏高原黄河流 域源头地区地势较为平坦、河流下切较弱，青藏高原 东部怒江、澜沧江和长江流域地区地势起伏度大、 河流下切较深(Liu-Zeng等, 2008), 雅鲁藏布江及东构 造结地区构造活动强烈、地势起伏度大、河流下切 较深(Zhang等, 2021), 剥蚀速率呈现与地形起伏、河 流下切和构造活动强度正相关关系(图6a). 然而, 异常 高的剥蚀速率总是来自于构造活动强烈地区，例如位 于鲜水河断裂带的贡嘎山流域剥蚀速率 $(>1000 \mathrm{~mm}$ $\mathrm{kyr}^{-1}, N=9$ )(Cook等，2018)和位于喜马拉雅东构造结 地区流域剥蚀速率 $\left(>1000 \mathrm{~mm} \mathrm{kyr}^{-1}, N=11\right)$ (Lupker等, 2017; Zhang等, 2021). 由于构造活动引起的地震和滑 坡同样可以造成异常高的流域剥蚀速率，例如在2008 年汶川地震后流域剥蚀速率的“增强”(West等，2014; Li等，2017; Wang等，2017), 所以在青藏高原等高 山、高起伏和河流下切较深地区异常高的流域剥蚀 速率很可能衍生于短期非稳态地表被低估宇宙成因 核素浓度的样品.一旦样品来自于深部或非稳态地区, 核素浓度非饱和状态的样品不满足流域剥蚀模型的 稳态假设，且往往会高估流域剥蚀速率。以稳态模型 得出高估的流域剥蚀速率可以用于对比研究区域的 地表稳定性, 但不能定量得到流域的长期稳定剥蚀 速率. 

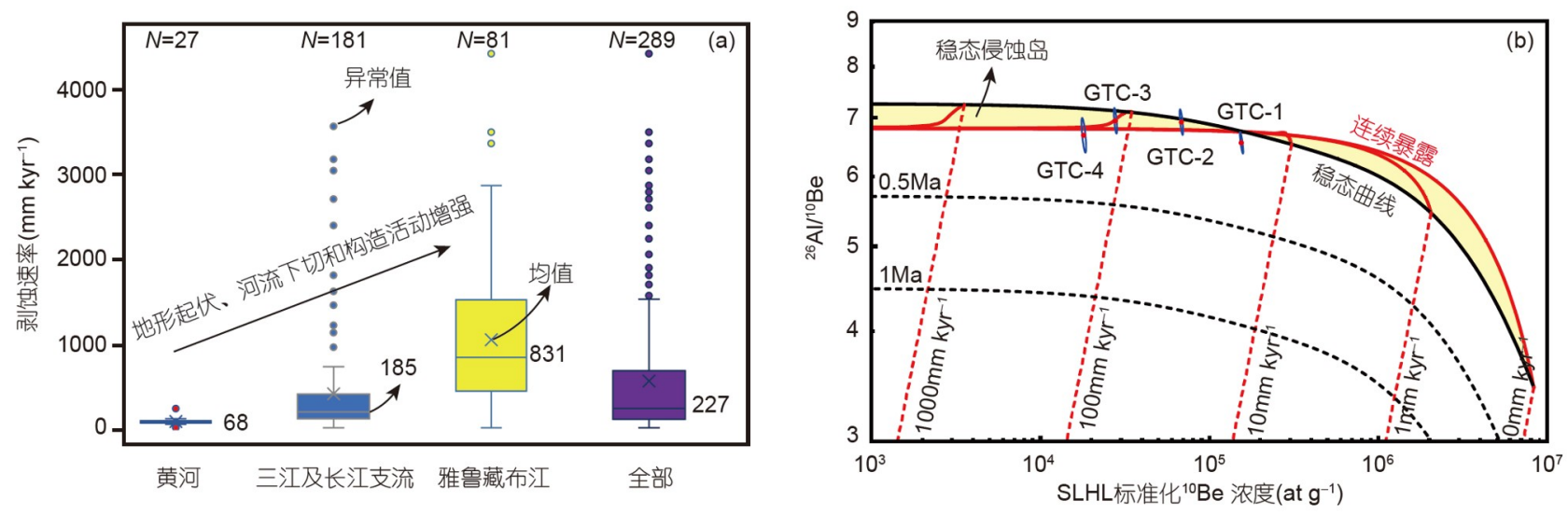

图 6 (a)青藏高原部分地区 ${ }^{10} \mathrm{Be}$ 流域剥蚀速率箱式图及(b) 腾冲剖面样品(GTC)宇宙成因 ${ }^{10} \mathrm{Be}^{26}{ }^{26} \mathrm{Al}$ 核素对图解(稳态侵蚀岛) (a) 其中三江及长江支流包括怒江、澜沧江、金沙江、雅聋江、大渡河和峎江, 一个异常高值 $\left(7600 \mathrm{~mm} \mathrm{kyr}^{-1}\right)$ 未显示在范围内. 图中 $N$ 代表样 品个数, 底部数字代表统计中值. 黄河、三江及长江支流数据汇总见Yang等(2019)附表3. 雅鲁藏布江数据引自Lupker等(2017)和Zhang等 (2021). 前人流域剥蚀速率均假设地表密度为 $2.70 \mathrm{~g} \mathrm{~cm}^{-3}$. (b) 其中 ${ }^{10} \mathrm{Be}$ 浓度根据Stone (2000)归一化到SLHL, 红色虚线为埋藏前流域剥蚀速率

在 ${ }^{26} \mathrm{Al}-{ }^{10} \mathrm{Be}$ 双核素稳态侵蚀岛图中(图6b), 腾冲剖 面样品均落在稳态侵蚀岛内. 一般认为, 这一结果显示 样品经历了连续暴露, 即无埋藏历史. 然而仅根据稳态 侵蚀岛图仍然无法判断样品是否真正处于稳态. 若腾 冲剖面经历快速剥蚀事件并将不同深度样品剥蚀至流 域中埋藏或暴露，埋藏或暴露前流域剥蚀速率与剥蚀 深度呈正相关(图6b), 且越深的样品(例如GTC-4)代表 了剥蚀速率越高，但并不能反映该流域的真实平均剥 蚀速率. 在地质历史长时间尺度上, 地形地貌演化过 程中很难处于长期稳定状态，尤其是在构造活动变化 强烈和气候变化明显地区. 深度剖面法可以为研究非 稳态地区地形地貌演化提供更为精确和有效的地表剥 蚀速率和暴露年龄. 根据野外观察、区域背景和模拟 结果拟合度可以选择使用剥蚀速率变化或短期剥蚀事 件模型, 例如长时间尺度气候变化(中国东部季风气候 影响地区及青藏高原内部构造活动稳定地区)和植被 类型改变明显地区使用剥蚀速率变化模型; 青藏高原 构造活动(喜马拉雅东构造结、青藏高原东南深切峡 谷和东部龙门山推覆构造带)和冰川剥蚀作用强烈地 区使用短期剥蚀事件模型。且两种非稳态模型在模拟 结果上也有相似的地貌演化指示意义, 例如蓟县剖面 剥蚀速率变化模型年龄 $10_{-8}^{+90} \mathrm{ka}$ 和后剥蚀速率 $214_{-164}^{+286}$ $\mathrm{mm} \mathrm{kyr}{ }^{-1}$ 最优值计算的剥蚀深度 $2140 \mathrm{~mm}$ 与短期剥蚀 事件 $0_{-0}^{+27.3} \mathrm{ka}$ 时经历 $177_{-77}^{+2323} \mathrm{~mm}$ 剥蚀深度在误差范围内 基本一致，表明剖面在千年时间尺度上发生了强烈的
地表剥蚀作用; 同样腾冲剖面两种模型均显示在 $\sim 157 \mathrm{ka}\left({ }^{10} \mathrm{Be}\right)$ 或 $127 \mathrm{ka}\left({ }^{26} \mathrm{Al}\right)$ 剖面可能经历了强烈的地 表剥蚀.

\section{5 结论}

本文对比了天津蓟县和云南腾冲两个典型地区花 岗岩风化壳深度剖面稳态和非稳态宇宙成因核素模 型, 结合前人对青藏高原地区流域剥蚀速率的研究结 果, 得到如下结论.

(1) 天津蓟县和云南腾冲花岗岩风化壳剖面均处 于非稳定状态.

(2) 非稳态模型表明蓟县剖面在千年时间尺度上 经历了剥蚀速率改变或地表剥蚀事件, 其对应的非稳 态模型结果 $14.6 \mathrm{~mm} \mathrm{kyr}^{-1}$ 可能真实地反映了地表的 长期剥蚀速率, 而夸大的剥蚀速率 $\left(239.0 \mathrm{~mm} \mathrm{kyr}^{-1}\right)$ 则 由于稳态前提假设的不合理所致.

(3) 腾冲剖面非稳态模型结果表明, 在 $157 \mathrm{ka}$ $\left({ }^{10} \mathrm{Be}\right)$ 或 $127 \mathrm{ka}\left({ }^{26} \mathrm{Al}\right)$ 剖面可能经历了强烈的地表剥蚀, 该剥蚀事件年龄与MIS 6冰期结束年龄 $(130 \mathrm{ka})$ 基本一 致, 可能反映青藏高原东南缘由冰期向间冰期转变过 程中对地表地貌的强烈改造作用.

（4）稳态假设条件下的剥蚀速率与区域构造活动 强度成正相关关系, 显示构造活动强烈的非稳态区域 宇宙成因核素剥蚀速率很可能由于稳态假设而被 高估. 


\section{参考文献}

杨业, 刘或, 马严, 徐胜, 刘丛强, 王世杰, Stuart F M, Fabel D. 2021 贵州高原沉积物原位宇宙成因核素 ${ }^{10} \mathrm{Be} 、{ }^{26} \mathrm{~A} 1$ 和 ${ }^{21} \mathrm{Ne}$ 测年研究. 中国科学: 地球科学, 51: 1275-1288

Ackerer J, Chabaux F, Van der Woerd J, Viville D, Pelt E, Kali E, Lerouge C, Ackerer P, di Chiara Roupert R, Négrel P. 2016. Regolith evolution on the millennial timescale from combined UTh-Ra isotopes and in situ cosmogenic ${ }^{10} \mathrm{Be}$ analysis in a weathering profile (Strengbach catchment, France). Earth Planet Sci Lett, 453: $33-43$

Ansberque C, Godard V, Bellier O, De Sigoyer J, Liu-Zeng J, Xu X, Ren Z, Li Y, ASTER Team. 2015. Denudation pattern across the Longriba fault system and implications for the geomorphological evolution of the eastern Tibetan margin. Geomorphology, 246: 542557

Balco G. 2017. Production rate calculations for cosmic-ray-muonproduced ${ }^{10} \mathrm{Be}$ and ${ }^{26} \mathrm{Al}$ benchmarked against geological calibration data. Quat Geochronol, 39: 150-173

Balco G, Stone J O, Lifton N A, Dunai T J. 2008. A complete and easily accessible means of calculating surface exposure ages or erosion rates from ${ }^{10} \mathrm{Be}$ and ${ }^{26} \mathrm{Al}$ measurements. Quat Geochronol, 3: 174 195

Blard P H, Lupker M, Rousseau M. 2019. Paired-cosmogenic nuclide paleoaltimetry. Earth Planet Sci Lett, 515: 271-282

Borchers B, Marrero S, Balco G, Caffee M, Goehring B, Lifton N, Nishiizumi K, Phillips F, Schaefer J, Stone J. 2016. Geological calibration of spallation production rates in the CRONUS-Earth project. Quat Geochronol, 31: 188-198

Braucher R, Brown E T, Bourlès D L, Colin F. 2003. In situ produced

${ }^{10} \mathrm{Be}$ measurements at great depths: Implications for production rates by fast muons. Earth Planet Sci Lett, 211: 251-258

Braucher R, Del Castillo P, Siame L, Hidy A J, Bourlés D L. 2009. Determination of both exposure time and denudation rate from an in situ produced ${ }^{10} \mathrm{Be}$ depth profile: A mathematical proof of uniqueness. Model sensitivity and applications to natural cases. Quat Geochronol, 4: 56-67

Braucher R, Bourlès D, Merchel S, Vidal Romani J, FernadezMosquera D, Marti K, Léanni L, Chauvet F, Arnold M, Aumaître G, Keddadouche K. 2013. Determination of muon attenuation lengths in depth profiles from in situ produced cosmogenic nuclides. Nucl Instrum Methods Phys Res Sect B-Beam Interact Mater Atoms, 294: 484-490

Charreau J, Saint-Carlier D, Dominguez S, Lavé J, Blard P H, Avouac J P, Jolivet M, Chen Y, Wang S L, Brown N D, Malatesta L C, Rhodes E. 2017. Denudation outpaced by crustal thickening in the eastern Tianshan. Earth Planet Sci Lett, 479: 179-191

Chevalier M L, Leloup P H, Replumaz A, Pan J, Métois M, Li H. 2017. Temporally constant slip rate along the Ganzi fault, NW Xianshuihe fault system, eastern Tibet. GSA Bull, 130: 396-410

Chevalier M L, Replumaz A. 2019. Deciphering old moraine age distributions in SE Tibet showing bimodal climatic signal for glaciations: Marine Isotope Stages 2 and 6. Earth Planet Sci Lett, 507: $105-118$

Chmeleff J, von Blanckenburg F, Kossert K, Jakob D. 2010. Determination of the ${ }^{10} \mathrm{Be}$ half-life by multicollector ICP-MS and liquid scintillation counting. Nucl Instrum Methods Phys Res Sect B-Beam Interact Mater Atoms, 268: 192-199

Clark P U, Dyke A S, Shakun J D, Carlson A E, Clark J, Wohlfarth B, Mitrovica J X, Hostetler S W, McCabe A M. 2009. The Last Glacial Maximum. Science, 325: 710-714

Cook K L, Hovius N, Wittmann H, Heimsath A M, Lee Y H. 2018. Causes of rapid uplift and exceptional topography of Gongga Shan on the eastern margin of the Tibetan Plateau. Earth Planet Sci Lett, 481: $328-337$

Cui L, Yang Y, Xu S, Zhao Z, Mao H, Zhang X, Tu C, Zhang Z, Liu W, Liu C. 2021. Denudation rates of granitic regolith along climatic gradient in Eastern China. Geomorphology, 390: 107872

Cui L F, Liu C Q, Xu S, Zhao Z Q, Liu T Z, Liu W J, Zhang Z J. 2016. Subtropical denudation rates of granitic regolith along a hill ridge in Longnan, SE China derived from cosmogenic nuclide depthprofiles. J Asian Earth Sci, 117: 146-152

Delmas M, Calvet M, Gunnell Y, Voinchet P, Manel C, Braucher R, Tissoux H, Bahain J J, Perrenoud C, Saos T. 2018. Terrestrial ${ }^{10} \mathrm{Be}$ and electron spin resonance dating of fluvial terraces quantifies quaternary tectonic uplift gradients in the eastern Pyrenees. Quat Sci Rev, 193: 188-211

Dong K, Lang Y, Hu N, Zhong J, Xu S, Hauser T M, Gan R. 2018. The new AMS facility at Tianjin University. Radiat Detect Technol Methods, 2: 30

Dunai T J. 2010. Cosmogenic Nuclides: Principles, Concepts and Applications in the Earth Surface Sciences. Cambridge: Cambridge University Press

Godard V, Lavé J, Carcaillet J, Cattin R, Bourlès D, Zhu J. 2010. Spatial distribution of denudation in Eastern Tibet and regressive erosion of plateau margins. Tectonophysics, 491: 253-274

Gosse J C, Phillips F M. 2001. Terrestrial in situ cosmogenic nuclides: Theory and application. Quat Sci Rev, 20: 1475-1560

Harkins N, Kirby E, Heimsath A, Robinson R, Reiser U. 2007. Transient fluvial incision in the headwaters of the Yellow River, northeastern Tibet, China. J Geophys Res, 112: F03S04

Heimsath A M, Dibiase R A, Whipple K X. 2012. Soil production limits 
and the transition to bedrock-dominated landscapes. Nat Geosci, 5: 210-214

Henck A C, Huntington K W, Stone J O, Montgomery D R, Hallet B. 2011. Spatial controls on erosion in the Three Rivers Region, southeastern Tibet and southwestern China. Earth Planet Sci Lett, 303: $71-83$

Hidy A J, Gosse J C, Pederson J L, Mattern J P, Finkel R C. 2010. A geologically constrained Monte Carlo approach to modeling exposure ages from profiles of cosmogenic nuclides: An example from Lees Ferry, Arizona. Geochem Geophys Geosyst, 11: Q0AA10

Kirby E, Harkins N. 2013. Distributed deformation around the eastern tip of the Kunlun fault. Int J Earth Sci-Geol Rund, 102: 1759-1772

Kohl C P, Nishiizumi K. 1992. Chemical isolation of quartz for measurement of in-situ -produced cosmogenic nuclides. Geochim Cosmochim Acta, 56: 3583-3587

Kong P, Granger D E, Wu F Y, Caffee M W, Wang Y J, Zhao X T, Zheng Y. 2009. Cosmogenic nuclide burial ages and provenance of the Xigeda paleo-lake: Implications for evolution of the Middle Yangtze River. Earth Planet Sci Lett, 278: 131-141

Korschinek G, Bergmaier A, Faestermann T, Gerstmann U C, Knie K, Rugel G, Wallner A, Dillmann I, Dollinger G, von Gostomski C L, Kossert K, Maiti M, Poutivtsev M, Remmert A. 2010. A new value for the half-life of ${ }^{10} \mathrm{Be}$ by heavy-ion elastic recoil detection and liquid scintillation counting. Nucl Instrum Methods Phys Res Sect B-Beam Interact Mater Atoms, 268: 187-191

Lal D. 1991. Cosmic ray labeling of erosion surfaces: In situ nuclide production rates and erosion models. Earth Planet Sci Lett, 104: 424-439

Li G, West A J, Densmore A L, Jin Z, Zhang F, Wang J, Clark M, Hilton R G. 2017. Earthquakes drive focused denudation along a tectonically active mountain front. Earth Planet Sci Lett, 472: 253265

Li N, Zhao Y W, Zhang L Y, Wang J L. 2020. The quaternary eruptive sequence of the Tengchong volcanic group, southwestern China. Lithos, 354-355: 105173

Lisiecki L E, Raymo M E. 2005. A Pliocene-Pleistocene stack of 57 globally distributed benthic $\delta^{18} \mathrm{O}$ records. Paleoceanography, 20: PA1003

Liu-Zeng J, Tapponnier P, Gaudemer Y, Ding L. 2008. Quantifying landscape differences across the Tibetan plateau: Implications for topographic relief evolution. J Geophys Res, 113: F04018

Lupker M, Lavé J, France-Lanord C, Christl M, Bourlès D, Carcaillet J, Maden C, Wieler R, Rahman M, Bezbaruah D, Xiaohan L. 2017. ${ }^{10} \mathrm{Be}$ systematics in the Tsangpo-Brahmaputra catchment: The cosmogenic nuclide legacy of the eastern Himalayan syntaxis.
Earth Surf Dynam, 5: 429-449

Marrero S M, Phillips F M, Borchers B, Lifton N, Aumer R, Balco G. 2016. Cosmogenic nuclide systematics and the CRONUScalc program. Quat Geochronol, 31: 160-187

Nishiizumi K. 2004. Preparation of ${ }^{26} \mathrm{Al}$ AMS standards. Nucl Instrum Methods Phys Res Sect B-Beam Interact Mater Atoms, 223-224: 388-392

Ouimet W B, Whipple K X, Granger D E. 2009. Beyond threshold hillslopes: Channel adjustment to base-level fall in tectonically active mountain ranges. Geology, 37: 579-582

Pan B, Su H, Hu Z, Hu X, Gao H, Li J, Kirby E. 2009. Evaluating the role of climate and tectonics during non-steady incision of the Yellow River: Evidence from a $1.24 \mathrm{Ma}$ terrace record near Lanzhou, China. Quat Sci Rev, 28: 3281-3290

Parnell A C, Buck C E, Doan T K. 2011. A review of statistical chronology models for high-resolution, proxy-based Holocene palaeoenvironmental reconstruction. Quat Sci Rev, 30: 29482960

Phillips F M, Argento D C, Balco G, Caffee M W, Clem J, Dunai T J, Finkel R, Goehring B, Gosse J C, Hudson A M, Jull A J T, Kelly M A, Kurz M, Lal D, Lifton N, Marrero S M, Nishiizumi K, Reedy R C, Schaefer J, Stone J O H, Swanson T, Zreda M G. 2016. The CRONUS-earth project: A synthesis. Quat Geochronol, 31: 119-154

Rodés Á, Evans D L. 2020. Cosmogenic soil production rate calculator. MethodsX, 7: 100753

Rodés Á, Pallàs R, Braucher R, Moreno X, Masana E, Bourlés D L. 2011. Effect of density uncertainties in cosmogenic ${ }^{10} \mathrm{Be}$ depthprofiles: Dating a cemented Pleistocene alluvial fan (Carboneras Fault, SE Iberia). Quat Geochronol, 6: 186-194

Shen G, Gao X, Gao B, Granger D E. 2009. Age of Zhoukoudian Homo erectus determined with ${ }^{26} \mathrm{Al} /{ }^{10} \mathrm{Be}$ burial dating. Nature, 458: 198 200

Shi X, Furlong K P, Kirby E, Meng K, Marrero S, Gosse J, Wang E, Phillips F. 2017. Evaluating the size and extent of paleolakes in central Tibet during the late Pleistocene. Geophys Res Lett, 44: $5476-5485$

Siame L, Bellier O, Braucher R, Sébrier M, Cushing M, Bourlès D, Hamelin B, Baroux E, de Voogd B, Raisbeck G, Yiou F. 2004. Local erosion rates versus active tectonics: Cosmic ray exposure modelling in Provence (south-east France). Earth Planet Sci Lett, 220: $345-364$

Stone J O. 2000. Air pressure and cosmogenic isotope production. J Geophys Res, 105: 23753-23759

Stone J O H, Evans J M, Fifield L K, Allan G L, Cresswell R G. 1998. Cosmogenic chlorine-36 production in calcite by muons. Geochim Cosmochim Acta, 62: 433-454 
Strasky S, Graf A A, Zhao Z, Kubik P W, Baur H, Schlüchter C, Wieler R. 2009. Late Glacial ice advances in southeast Tibet. J Asian Earth Sci, 34: 458-465

Tao Y, Xiong J, Zhang H, Chang H, Li L. 2020. Climate-driven formation of fluvial terraces across the Tibetan Plateau since $200 \mathrm{ka}$ : A review. Quat Sci Rev, 237: 106303

Thompson L G, Yao T, Davis M E, Henderson K A, Mosley-Thompson E, Lin P N, Beer J, Synal H A, Cole-Dai J, Bolzan J F. 1997. Tropical climate instability: The last glacial cycle from a QinghaiTibetan ice core. Science, 276: 1821-1825

Wang W, Godard V, Liu-Zeng J, Scherler D, Xu C, Zhang J, Xie K, Bellier O, Ansberque C, de Sigoyer J. 2017. Perturbation of fluvial sediment fluxes following the 2008 Wenchuan earthquake. Earth Surf Proc Land, 42: 2611-2622

West A J, Hetzel R, Li G, Jin Z, Zhang F, Hilton R G, Densmore A L. 2014. Dilution of ${ }^{10} \mathrm{Be}$ in detrital quartz by earthquake-induced landslides: Implications for determining denudation rates and potential to provide insights into landslide sediment dynamics. Earth Planet Sci Lett, 396: 143-153

Wolkowinsky A J, Granger D E. 2004. Early Pleistocene incision of the San Juan River, Utah, dated with ${ }^{26} \mathrm{Al}$ and ${ }^{10} \mathrm{Be}$. Geology, 32: 749752

Xu S, Dougans A B, Freeman S P H T, Schnabel C, Wilcken K M.
2010. Improved ${ }^{10} \mathrm{Be}$ and ${ }^{26} \mathrm{Al}-\mathrm{AMS}$ with a $5 \mathrm{MV}$ spectrometer. Nucl Instrum Methods Phys Res Sect B-Beam Interact Mater Atoms, 268: 736-738

Yang Y, Lang Y C, Xu S, Liu C Q, Cui L F, Freeman S P H T, Wilcken K M. 2020. Combined unsteady denudation and climatic gradient factors constrain carbonate landscape evolution: New insights from in situ cosmogenic ${ }^{36} \mathrm{Cl}$. Quat Geochronol, 58: 101075

Yang Y, Liu C Q, Van der Woerd J, Xu S, Cui L F, Zhao Z Q, Wang Q L, Jia G D, Chabaux F. 2019. New constraints on the late Quaternary landscape evolution of the eastern Tibetan Plateau from ${ }^{10} \mathrm{Be}$ and ${ }^{26} \mathrm{Al}$ in-situ cosmogenic nuclides. Quat Sci Rev, 220: 244 262

Zhang X L, Cui L F, Xu S, Liu C Q, Zhao Z Q, Zhang M L, Liu-Zeng J. 2021. Assessing non-steady-state erosion processes using paired ${ }^{10} \mathrm{Be}-{ }^{26} \mathrm{Al}$ in southeastern Tibet. Earth Surf Proc Land, 46: 13631374

Zhou J, Zhou W, Dong G, Hou Y, Xian F, Zhang L, Tang L, Zhao G, Fu Y. 2020. Cosmogenic ${ }^{10} \mathrm{Be}$ and ${ }^{26} \mathrm{Al}$ exposure dating of Nam Co lake terraces since MIS 5, southern Tibetan Plateau. Quat Sci Rev, 231: 106175

Zhou S, Wang J, Xu L, Wang X, Colgan P M, Mickelson D M. 2010. Glacial advances in southeastern Tibet during late Quaternary and their implications for climatic changes. Quat Int, 218: 58-66

(责任编委: 胡建芳) 